\title{
INEQUALITIES INVOLVING THE INTEGRALS OF POLYNOMIALS AND THEIR POLAR DERIVATIVES
}

\author{
N. A. Rather, Suhail Gulzar and S. H. Ahangar
}

Abstract. For the class of polynomials $P(z)$ of degree $n$ having all their zeros in $|z| \leqslant k$ where $k \leqslant 1$, Aziz [1] proved that for each $q>0$,

$$
n\left\{\int_{0}^{2 \pi}\left|\frac{P\left(e^{i \theta}\right)}{P^{\prime}\left(e^{i \theta}\right)}\right|^{q} d \theta\right\}^{1 / q} \leqslant\left\{\int_{0}^{2 \pi}\left|k+e^{i \theta}\right|^{q} d \theta\right\}^{1 / q} .
$$

In this paper, we extend this inequality to the polar derivative in the sense that we take the polar derivative $D_{\alpha} P(z)$ in place of ordinary derivative $P^{\prime}(z)$ of polynomial $P(z)$. We also obtain analogous inequalities for the class of lacunary polynomials $P(z)=a_{n} z^{n}+\sum_{j=\mu}^{n} a_{n-j} z^{n-j}, 1 \leqslant$ $\mu \leqslant n$, of degree $n$ having all their zeros in $|z| \leqslant k, k \leqslant 1$.

Mathematics subject classification (2010): 30C10, 30A10, 41A17.

Keywords and phrases: Polynomials, inequalities in the complex domain, polar derivative.

\section{REFERENCES}

[1] A. AzIZ, Integral mean estimates polynomials with restricted zeros, J. Approx. Theory, 55, (1988), 232-239.

[2] A. AZIZ AND N. A. RATHER, A refinement of a theorem of Paul Turan concerning polynomials, Math Ineq. Appl., 1, (1998) 231-238.

[3] A. AZIZ AND N. A. RATHER, Inequalities for the polar derivative of a polynomial with restricted zeros, Math. Balk., 17 (2003), 15-28.

[4] E. Hille, Analytic function theory, Vol. II, Ginn and Company, New York, Toronto, 1962.

[5] M. A. MaLiK, On the derivative of a polynomial, J. Lond. Math. Soc., 1 (1969), 57-60.

[6] M. A. Malik, An integral mean estimates for polynomials, Proc. Amer. Math. Soc., 91 (1984), 281284.

[7] M. Marden, Geometry of Polynomials, Math. Surveys no. 3, Amer. Math. Soc. Providence R. I. 1966.

[8] N. A. RATHER, Extremal properties and Location of the zeros of polynomials, $\mathrm{Ph} . \mathrm{D}$. Thesis, University of Kashmir, 1998.

[9] N. A. Rather, Faroz A. Bhat And Suhail Gulzar, Some $L_{r}$ inequalities involving the polar derivative of a polynomial, Appl. Math. E-Notes, 14 (2014), 116-122.

[10] P. TuRán, Uber die Ableitung von Polynomen, Compos. Math., 7 (1939), 89-95. 